ARTICLE

DOI: $10.1038 / \mathrm{s} 41467-018-03439-\mathrm{x}$

\title{
Label-free analysis of physiological hyaluronan size distribution with a solid-state nanopore sensor
}

\author{
Felipe Rivas ${ }^{1}$, Osama K. Zahid ${ }^{1}$, Heidi L. Reesink ${ }^{2}$, Bridgette T. Peal ${ }^{2}$, Alan J. Nixon², Paul L. DeAngelis ${ }^{3}$, \\ Aleksander Skardal $\mathbb{1}^{1,4,5}$, Elaheh Rahbar ${ }^{1} \&$ Adam R. Hall ${ }^{1,4,5}$
}

Hyaluronan (or hyaluronic acid, HA) is a ubiquitous molecule that plays critical roles in numerous physiological functions in vivo, including tissue hydration, inflammation, and joint lubrication. Both the abundance and size distribution of HA in biological fluids are recognized as robust indicators of various pathologies and disease progressions. However, such analyses remain challenging because conventional methods are not sufficiently sensitive, have limited dynamic range, and/or are only semi-quantitative. Here we demonstrate label-free detection and molecular weight discrimination of HA with a solid-state nanopore sensor. We first employ synthetic HA polymers to validate the measurement approach and then use the platform to determine the size distribution of as little as $10 \mathrm{ng}$ of HA extracted directly from synovial fluid in an equine model of osteoarthritis. Our results establish a quantitative method for assessment of a significant molecular biomarker that bridges a gap in the current state of the art.

\footnotetext{
${ }^{1}$ Virginia Tech-Wake Forest University School of Biomedical Engineering and Sciences, Wake Forest School of Medicine, Winston-Salem, NC 27101, USA. ${ }^{2}$ Department of Clinical Sciences, College of Veterinary Medicine, Cornell University, Ithaca, NY 14853, USA. ${ }^{3}$ Department of Biochemistry and Molecular Biology, University of Oklahoma Health Sciences Center, Oklahoma, OK 73104, USA. ${ }^{4}$ Institute for Regenerative Medicine, Wake Forest School of Medicine, Winston-Salem, NC 27101, USA. ${ }^{5}$ Comprehensive Cancer Center, Wake Forest School of Medicine, Winston-Salem, NC 27157, USA. Correspondence and requests for materials should be addressed to E.R. (email: erahbar@wakehealth.edu) or to A.R.H. (email: arhall@wakehealth.edu)
} 
yaluronan (or hyaluronic acid, $\mathrm{HA})^{1}$ is a polyanionic linear chain in the glycosaminoglycan (GAG) family featuring the alternating disaccharide repeat structure [-4D-glucuronic acid- $\beta 1-3-N$-acetylglucosamine- $\beta 1-]_{n}$. Distributed widely throughout mammalian cells and tissues, the biomechanical and biochemical properties of HA support its involvement in myriad physiological functions, including hydration and turgidity maintenance of tissue ${ }^{1}$, extracellular matrix structure ${ }^{1}$, regulation of innate immunity ${ }^{2}$, and protection and lubrication of joints ${ }^{3}$. As a result of this versatility, HA is considered a promising bioindicator of pathophysiology and inflammation, and has consequently been targeted for disease-specific diagnostics ${ }^{4,5}$. While the molecular weight (MW) of naturally occurring HA is typically ${ }^{6}$ in the range of $10^{5}-10^{7} \mathrm{Da}(\sim 250-25,000$ disaccharide units, each $\sim 1 \mathrm{~nm}$ in length), its size within this range is a critical determinant of the molecule's function in vivo. For example, high-MW HA $(>1000 \mathrm{kDa})$ is highly viscous and appears to display anti-inflammatory and immunosuppressive properties ${ }^{7}$; whereas, low-MW HA (generally $<500 \mathrm{kDa}$ ) can induce the release of pro-inflammatory cytokines from macrophages 8,9 . Furthermore, high-MW HA is far more responsible than lowMW HA for the lubricating properties of synovial fluid. Consequently, both the abundance and size distribution of HA are important biomarkers for disease pathologies and are essential to understanding the immunomodulatory and joint lubrication roles of HA in vivo.

Unfortunately, current technologies for HA detection and size differentiation have significant limitations. For example, techniques similar to enzyme-linked immunosorbent assays ${ }^{10,11}$ (ELISA) can be used for quantitative detection of HA, but have limited capacity to differentiate by MW and in some formats can neglect low-MW species. Supplementation of the approach with fractionation methods, such as size exclusion chromatography (SEC) enables discrete size ranges of HA to be quantified, but the nature of SEC (including slow column flow rates and long run times) places practical constraints on the number of fractions and samples that can be examined. Conversely, multi-angle laser light scattering (MALLS ${ }^{12}$ ) can report on HA MW, but is not intrinsically quantitative, has limited precision, and is relatively insensitive to low-MW fragments. Mass spectrometry (MS) ${ }^{13-15}$ is also capable of resolving MW differences, but in addition to requiring expensive and complex instrumentation, cannot probe HA larger than $\sim 100 \mathrm{kDa}$. As a result of these considerations, the most widely used HA assessment approach is agarose or polyacrylamide gel electrophoresis ${ }^{16,17}$, through which band intensity and position can be analyzed to denote a size distribution. However, this method is slow, requires large sample size (fluid volume and HA mass), requires calibrated standards (e.g., a

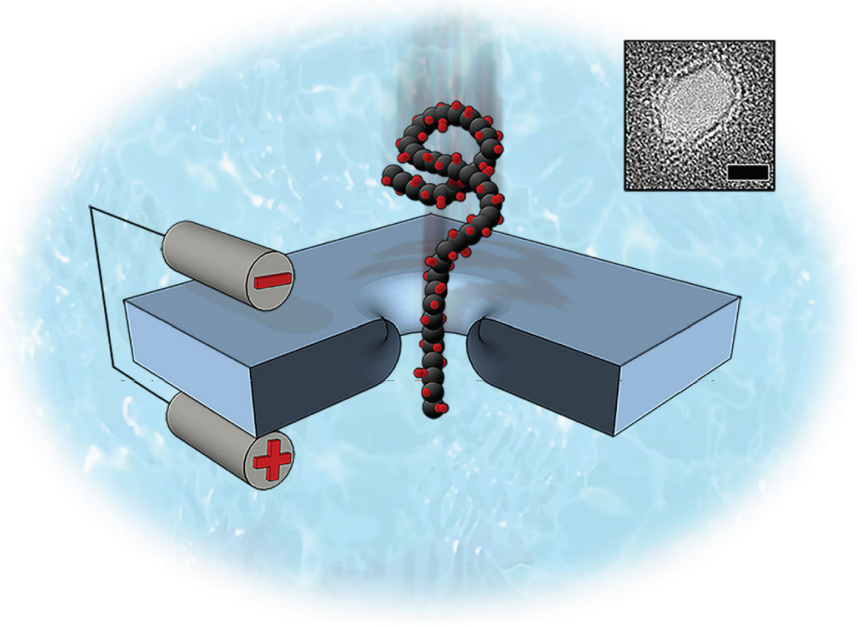

b

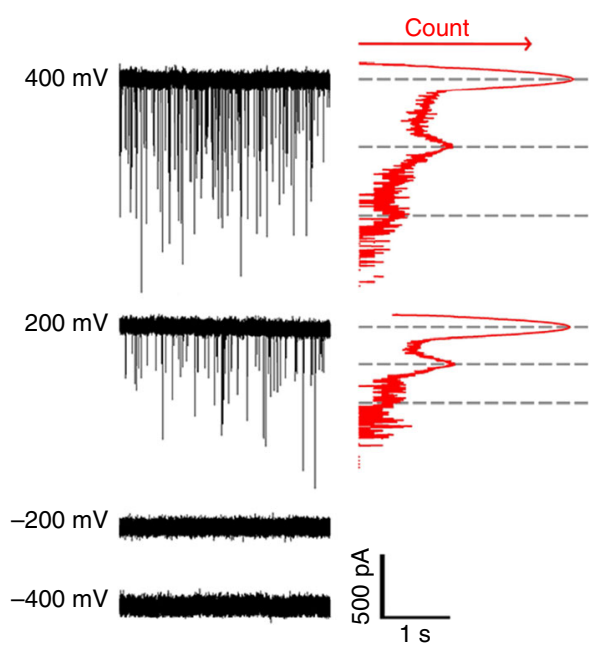

C

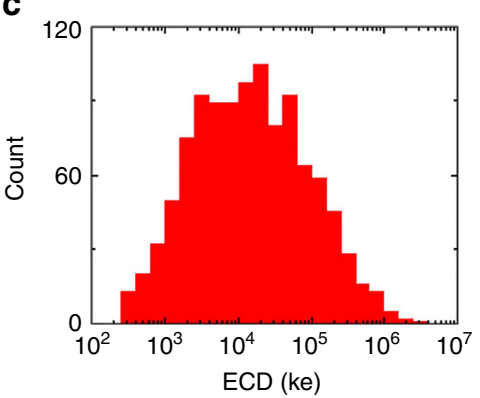

d

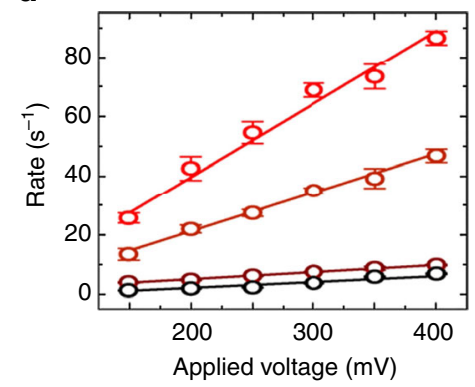

e

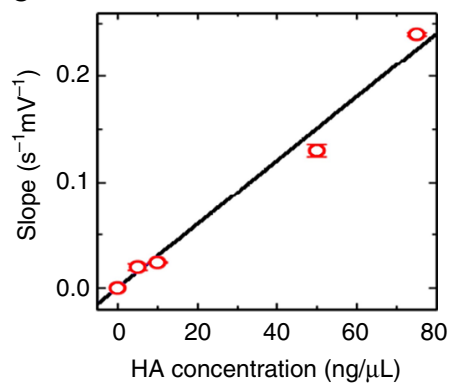

Fig. 1 SS-nanopore detection of polydisperse HA. a Schematic representation of electrophoretic translocation of HA through a SS-nanopore. Inset: transmission electromicrograph of a typical SS-nanopore fabricated with the same procedure used here. Scale bar, $5 \mathrm{~nm}$. b Raw current traces obtained from a $6.5 \mathrm{~nm}$ SS-nanopore with HA introduced on one chamber (cis-) and indicated voltage applied to the other (trans-). Events were observed only toward positive bias. All-points histograms (red) show quantized current levels (dashed lines), indicating molecular folding. c Typical ECD histogram for polydisperse HA $(n=1067)$ measured at $200 \mathrm{mV}$. d Voltage-dependent event rate for three concentrations of polydisperse HA ( $t$ - $b$ : 75, 50, 10, and $5 \mathrm{ng}$ / $\mu l) . N$-values (number of uninterrupted current traces) are listed in Supplementary Table 3 and error bars are standard deviations. Solid lines are linear fits to the data points. e Slopes from $\mathbf{d}$ showing a linear dependence (solid line) on net HA concentration. Error bars are errors of the fits 
a

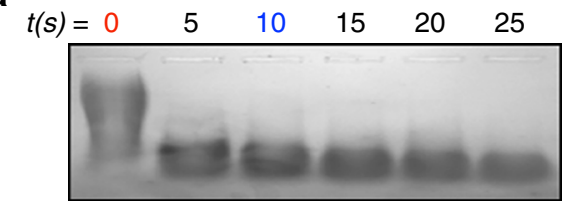

b
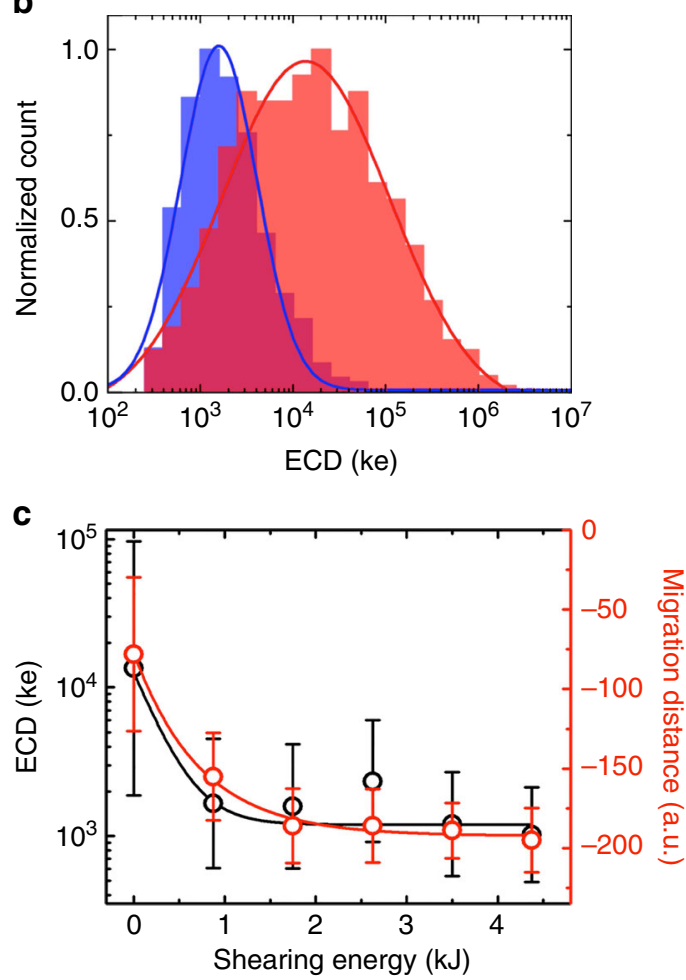

Fig. 2 Assessment of HA mechanical shearing. a Gel image of polydisperse $\mathrm{HA}$ under increasing durations of exposure to a $175 \mathrm{~W}$ ultrasonic shearing bath, demonstrating increasing fragmentation. $\mathbf{b}$ ECD histograms obtained from the $0 \mathrm{~s}$ (red) and $10 \mathrm{~s}$ (blue) samples from a. Solid lines are lognormal fits (Gaussian on a log scale) to the data. We observe a shift in the mean from $1.3 \times 10^{4} \mathrm{ke}$ (red) to $1.6 \times 10^{3} \mathrm{ke}$ (blue) after shearing with population width (standard deviation) reduced from $9.4 \times 10^{4} \mathrm{ke}(\mathrm{red})$ to $3.5 \times 10^{3} \mathrm{ke}$ (blue). c HA ECD (left, black) and gel migration distance (right, red) for all investigated shearing conditions, yielding nearly identical trends. Solid lines are exponential fits to the respective data. In b, c ECD data consist of $t=0$ ( $n=1067$, same data as Fig. 1c), $5(n=6331), 10 \quad(n=$ $1247), 15(n=617), 20(n=1008)$, and $25 \mathrm{~s}(n=781)$ and error bars represent the standard deviations of log-normal fits to the datasets

measured via MALLS or MS), and provides only semiquantitative data.

Solid-state (SS-) nanopores ${ }^{18,19}$ are an emerging platform for sensitive molecular analysis. The system uses a nanometer-scale aperture in a thin membrane (Fig. 1a, inset), positioned as the only fluid connection between two reservoirs of an electrolyte solution. An applied voltage is used to generate a strong electric field inside the opening that impels charged molecules electrophoretically through the pore and into the opposing chamber (Fig. 1a). During their residence inside the nanopore, each molecule occupies space that would otherwise be occupied by ions contributing to the electrical signal, and so their passage is marked by a temporary reduction (an "event") in the measured transmembrane ionic current. Analysis of the amplitude and the duration of events typically reports on the diameter and the contour length of the translocating molecules, respectively. The concept of resistive pulse sensing was first applied to HA by
Fennouri, et al. ${ }^{20,21}$, using the aerolysin protein pore. However, the dynamic range of that system is very narrow; direct assessment was limited to small HA ( $<10$ individual sugar residues) with only indirect evidence of larger molecule detection.

Here we establish the utility of fabricated SS-nanopores as a quantitative analytical tool for assessing HA. We first use synthetic HA to demonstrate that polysaccharides can be probed directly with the platform and to suggest the size-dependent nature of the measurement approach. We then employ HA populations with narrow size distributions to show that MW can be determined on a per molecule basis from the translocation signal. Finally, we demonstrate that our SS-nanopore approach can report on the size distribution of physiological HA isolated from the synovial fluid of an equine model of osteoarthritis (OA). The flexibility of our platform enables both detection and MW discrimination across a broad range of molecular sizes and its speed and quantitative output indicate a direct route to translational applications.

\section{Results}

SS-nanopore measurement of polydisperse HA. As an initial assessment of the utility of SS-nanopores to probe HA, we first conducted a set of experiments using a polydisperse (i.e., broad MW distribution) mixture of HA isolated from Streptococcus zooepidemicus fermentation (Methods section). The resulting current traces (Fig. 1b) confirmed the ability of SS-nanopores to resolve HA easily, typically yielding events that were at least five standard deviations $(5 \sigma)$ above the noise floor. As a negatively charged molecule (surface charge density of $-0.32 \mathrm{C} / \mathrm{m}^{2}$, with a low-isoelectric point ${ }^{22}$ of 2.5), HA was observed to move only toward the positive bias, indicating that its translocation was governed predominantly by electrophoresis ${ }^{23}$. Additionally, by reversing the applied bias after a measurement, we measured recaptured HA events (Supplementary Figure 1), confirming that the molecules fully translocated through the pore ${ }^{24}$. Turning to event characteristics, we noted integral variation in the measured translocation event depth histograms (Fig. 1b, right) that were suggestive of stochastic variations in molecular folding conformation during threading (Supplementary Figure 2), similar to past measurements with $\mathrm{DNA}^{25,26}$. While event durations have typically been more correlated with MW than depth in previous reports ${ }^{27}$, signal variations of this kind could skew the data, since folded molecules translocate more rapidly than unfolded ones. Consequently, we utilized for our analyses the experimental factor of event charge deficit (ECD) ${ }^{28}$, or integrated area defined by each event, such that a lower ECD corresponds to a lower MW HA chain. We chose this value because it comprises both event amplitude and duration, and thereby normalized potential differences in molecular conformation. Considering only event duration (Supplementary Figure 3), a folded molecule would appear smaller than an unfolded molecule of the same length. For our data, a typical polydisperse HA ECD histogram (Fig. 1c) showed a log-normal distribution (i.e., Gaussian on a log scale) spanning over four orders of magnitude. This wide population was indicative of the broad MW distribution within the sample.

Further probing the translocation dynamics of polydisperse HA through SS-nanopores, we measured the dependence of molecular capture rate on both applied voltage and net sample concentration (Fig. 1d). For all measured conditions, we observed a linear relationship between voltage and event rate, indicating a diffusion-limited translocation regime ${ }^{29}$ and suggesting that there was no significant energetic barrier related to entry of HA into the confined space of the nanopore for our system ${ }^{30}$. Crucially, another consequence of diffusion-limited kinetics is an absence of size dependence in event rate ${ }^{29}$, enabling an unbiased 
representation of MW distribution in the SS-nanopore signal. We also observed that event rates were strongly impacted by the net concentration of polydisperse $\mathrm{HA}$ in solution. Measurements yielded a linear response in recorded event rate dependence (slope) between 5 and $75 \mathrm{ng} / \mu \mathrm{l}$ (Fig. 1e). Featuring an intercept at 0 , this result suggested that arbitrarily low concentrations could in principle be probed with a concomitant reduction in measured event rate. Furthermore, translocations could also be detected above $75 \mathrm{ng} / \mu \mathrm{l}$, but often caused clogging at high-applied voltages, and therefore were not included here. Taken as a whole, this predictable variation indicated a route toward direct quantification of total HA with SS-nanopores, similar to previous studies on nucleic acids ${ }^{31}$ and nucleoprotein-protein complexes $^{32}$.

A critical objective of our analysis was MW discrimination. As an initial test to demonstrate the ability of SS-nanopores to resolve differences in HA size, we first used ultrasonic shearing to fragment the same polydisperse material artificially. Separate aliquots of polydisperse HA were mechanically sheared using constant ultrasonication energy across a range of time durations, such that treated HA chains would be reduced in size to increasingly smaller chain lengths. The samples were first examined by agarose gel electrophoresis (Fig. 2a), showing both a reduced population width and a greater migration distance as shearing power was increased, thereby indicating narrowing distributions with smaller mean MW. This material was subsequently measured by the SS-nanopore. ECD distributions (Fig. 2b and Supplementary Figure 4) for the untreated control and a representative sheared sample $(t=10 \mathrm{~s}$, corresponding to $\sim 1.8 \mathrm{~kJ}$ shearing energy) agreed qualitatively with gel observations, showing a narrower distribution and a clear shift toward lower ECD. Indeed, a comparison of ECD distributions (Supplementary Table 1) with image analysis of the gel across shearing conditions demonstrated remarkable agreement between the two independent datasets (Fig. 2c), and suggested a straightforward correlation between $\mathrm{HA} \mathrm{MW}$ and measured ECD from the SS-nanopore.

MW discrimination with quasi-monodisperse HA. Having demonstrated HA detection with SS-nanopores with an initial validation of the size dependence of the approach, we next pursued direct MW discrimination by examining quasimonodisperse (i.e., very narrow size distribution approaching the ideal of a single MW) HA. For these studies, discrete samples of $\mathrm{HA}$ ranging in $\mathrm{MW}$ from $54 \mathrm{kDa}$ to $2.4 \mathrm{MDa}$ were produced via an established synthetic polymerization method ${ }^{33}$ yielding HA typically within $\pm 5 \%$ of mean $\mathrm{MW}$, as confirmed by gel electrophoresis (Fig. 3a). Lane intensity analysis (Fig. 3b) showed discrete populations for the set of quasi-monodisperse HA, demonstrating the experimental precision achievable by gel. Similarly, we observed a series of defined peaks in the measured ECD (Fig. 3c) upon probing the same materials individually by SS-nanopore. The population for each quasi-monodisperse peak was considerably narrower than that measured for polydisperse HA (c.f. Figure 1c). Indeed, this narrowness suggested a higher resolution for the nanopore sensor than for gel analysis. We found that ECD peak separations reduced for lower MW samples, but were distinguishable down to $\sim 80 \mathrm{kDa}$ under our conditions. For the largest samples (1.1 and 2.4 MDa), we also observed some low ECD background signal that we attributed to fragmentation during handling or storage. Notably, a similar background was also visible on gel in the form of a smear in those two lanes (Fig. 3a), further supporting the validity of our measurements.

Plotting the mean ECD for all quasi-monodisperse HA samples, we found regular variation with respect to MW across
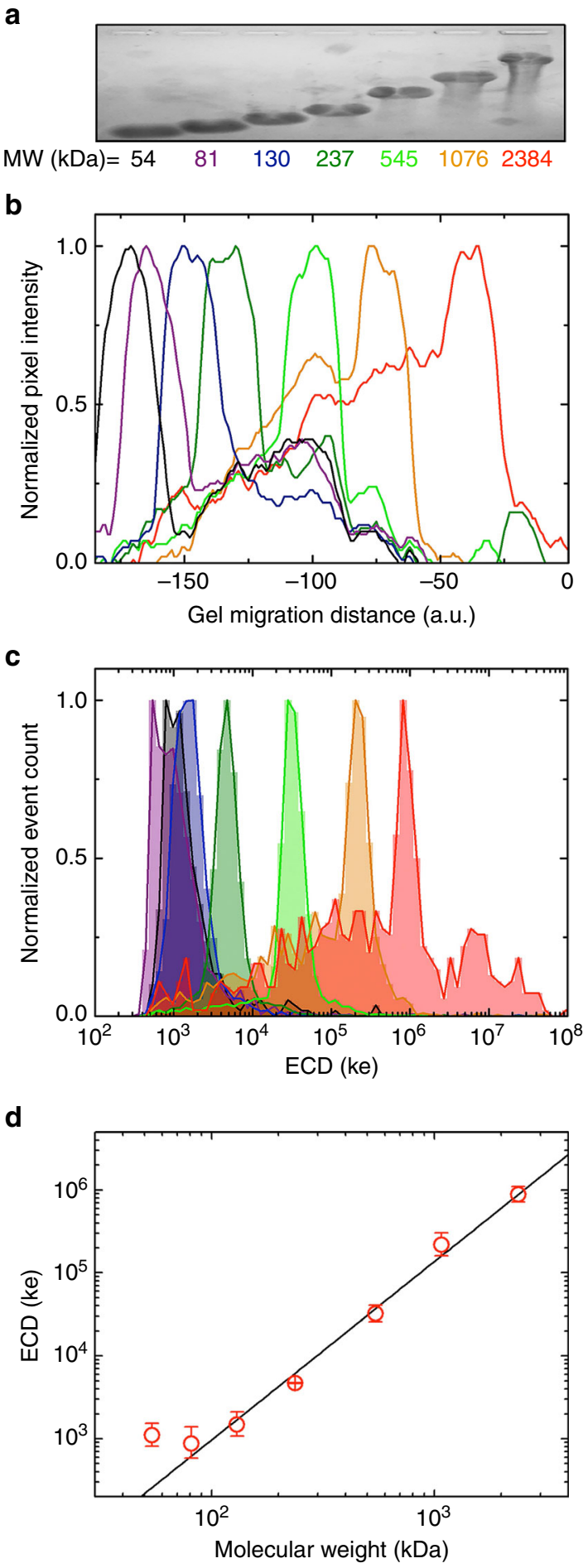

Fig. 3 SS-nanopore analysis of quasi-monodisperse HA. a Gel image of quasi-monodisperse HA samples. b Normalized electropherogram of gel image intensity for each MW sample. Colors match MW labels used in a. c ECD histograms for each MW sample measured at $200 \mathrm{mV}$ applied voltage, with number of events considered: $54(n=344), 81(n=1031)$, $130(n=3667), 237(n=7835), 545$ ( $n=5012), 1076(n=1743)$, and $2384 \mathrm{kDa}(n=640)$. Colors match MW labels used in a. d Relationship between ECD measured by SS-nanopore and HA MW from c. Error bars represent the standard deviations of log-normal fits to the datasets. Solid line is a power law fit ( $\alpha=2.23$ ) to the data down to $81 \mathrm{kDa}$ (see Supplementary Table 1 for fit details) 
a
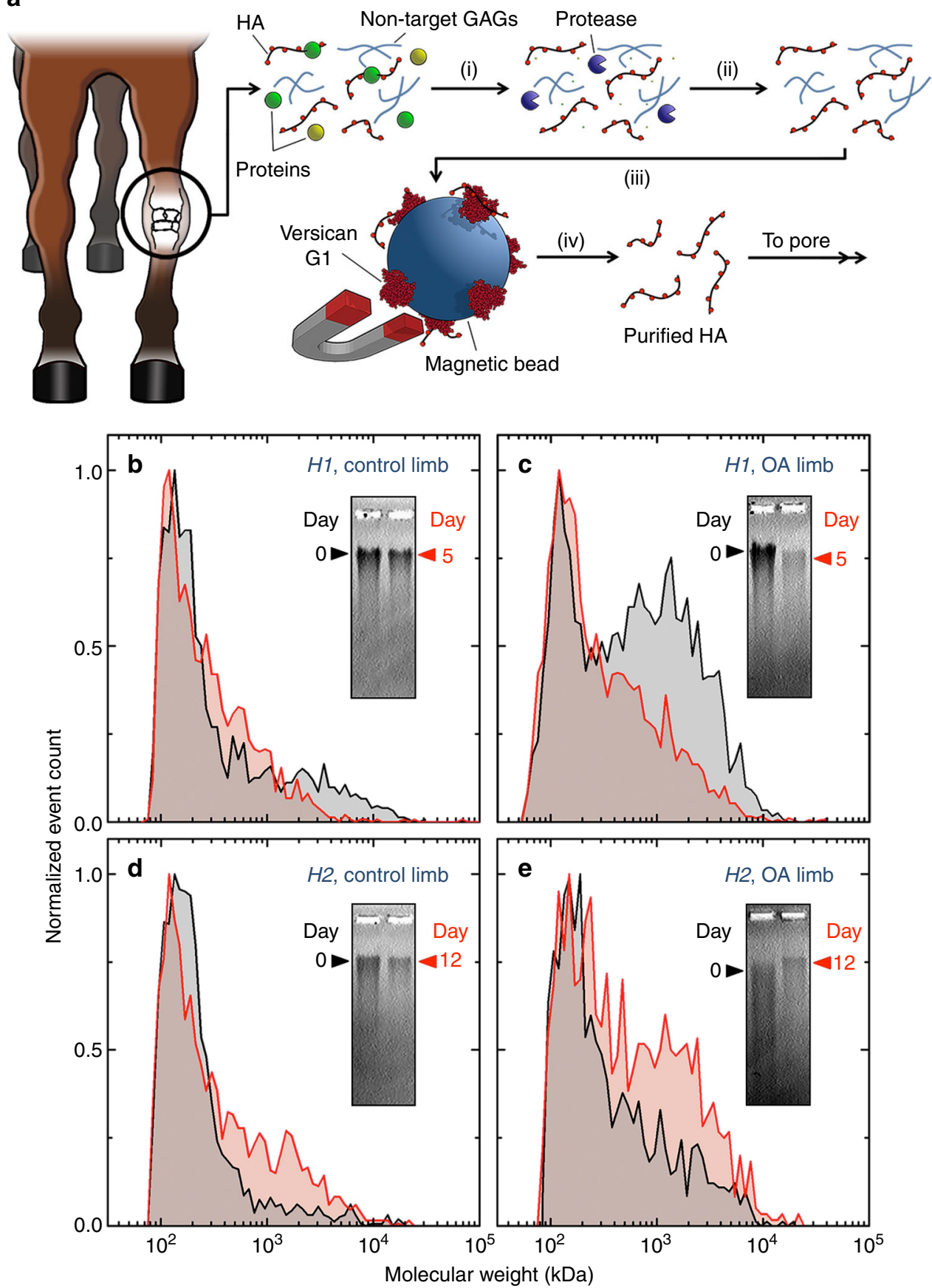

Fig. 4 Translational analysis of HA derived from equine synovial fluid. a Illustration of HA isolation protocol: (i) collected equine synovial fluid is treated with a broad-spectrum protease to digest proteins; (ii) liquid-liquid phase extraction is used to remove protease and remnant protein components; (iii) $\mathrm{HA}$ is selectively isolated on versican G1 magnetic beads; and (iv) elution yields pure HA for SS-nanopore analysis. b-e Equine synovial fluid HA size distributions obtained from SS-nanopore ECD analysis. For each, day 0 is black and day 5 or 12 is red. Horse $H 1$ control (sham knee): day 0 ( $n=1768$ ), day $5(n=1680)$; H1-induced OA knee: day O $(n=2590)$, day $5(n=2849) ; H 2$ control (sham knee): day O $(n=1748)$, day $12(n=1692) ; H 2$-induced OA knee: day $0(n=1141)$, day $12(n=1215)$. Measurements were performed on four different nanopores with diameters ranging from 7.6 to $9.6 \mathrm{~nm}$. Insets show accompanying gel images for the same synovial fluid samples with band positions marked

voltages (Fig. 3d and Supplementary Figure 5), well described over nearly the entire investigated range by a power law fit (Supplementary Table 1) yielding an average exponent $\alpha$ of 2.33 \pm 0.16 . Only the smallest sample $(54 \mathrm{kDa})$ deviated significantly from this relationship, possibly reflecting the time resolution limits of our current electronics. We expect that HA size differentiation at low-MW ranges could be improved, for example, through the use of high-bandwidth measurement techniques ${ }^{34}$. The observed power law trend was similar to length dependences measured for other biopolymer translocation durations through SS-nanopores, and was again indicative of the impact of diffusion-limited kinetics. We note that the exponent recovered from our fits (2.33) was higher than previous reports for double-strand $\mathrm{DNA}^{27,31}$, which ranged from 1.05 to 1.27 . This difference could be a result of increased diffusion facilitated by the more compact entropic conformation of $\mathrm{HA}$ and reduced 
self-avoidance in our high-ionic strength conditions ${ }^{35}$. Regardless, establishment of this trend provided a critical conversion, enabling determination of HA MW at the single-molecule level from the direct electrical output of the SS-nanopore system; for example, using the established relationship as a standard curve, we could determine that the polydisperse HA sample (Fig. 1c) had a mean MW of $311 \pm 5 \mathrm{kDa}$ with an interquartile range from about $200-654 \mathrm{kDa}$.

Assessment of HA extracted from synovial fluid. We next applied our approach to the analysis of HA in physiological fluids. Here we focused on synovial fluid, where HA is the major viscoelastic component supporting joint lubrication and hydration ${ }^{3}$ and its degradation has been implicated in joint disease. For example, a reduction in HA size and concentration has been associated with $\mathrm{OA}^{36}$, a common joint pathology that leads to cartilage deterioration. This trend is critical because the viscoelastic and immunomodulatory functions of HA are sizedependent $^{7}$ : an observation that positions HA MW distribution in particular as a potentially valuable bioindicator of OA initiation, progression, and treatment efficacy ${ }^{37}$. However, because of the non-selective nature of SS-nanopore signals (i.e., any translocating macromolecule can produce an event), it was not possible to probe synovial fluid without processing to remove other spurious components of biological origin ${ }^{38}$. Therefore, we implemented a procedure (Fig. 4a) for HA isolation that took advantage of the high-binding specificity of the versican protein G1 domain for $\mathrm{HA}^{39}$.

In our procedure, we first used a broad-spectrum protease to digest protein components of physiological fluid (Fig. 4a, i), including lubricin, collagenases, and especially endogenous HAbinding proteins ${ }^{40}$ that could otherwise be retained in the collection scheme. Next, we removed remaining protein (including the exogenously added protease) and lipid components by liquid-liquid phase extraction (Fig. 4a, ii), leaving in solution HA and other aqueous components such as sulfated GAGs ${ }^{41}$. We then incubated the processed mixture with the versican G1 domain immobilized on superparamagnetic beads, followed by magnetic isolation and washing of excess material (Fig. 4a, iii). Finally, bound HA was eluted from the beads thermally (Fig. 4a, iv) to yield a sample suitable for subsequent SS-nanopore analysis. The full protocol typically produced $\sim 150 \mathrm{ng}$ of highpurity HA from $50 \mu$ of raw synovial fluid (Supplementary Table 2).

To test the feasibility and diagnostic potential of the SSnanopore system, we applied this HA isolation protocol to synovial fluid biospecimens from an established equine model of post-traumatic $\mathrm{OA}^{42}$ (see Methods section for details). For our initial demonstration of translational SS-nanopore analysis, we focused on two horses (Supplementary Figure 6). For the first (H1), conventional gel analysis (Fig. $4 \mathrm{~b}, \mathrm{c}$, insets) showed a shift in the HA population toward lower MW 5 days after surgical carpal chip induction of OA. This shift is generally indicative of HA degradation, accumulation of low-MW HA fragments, and disease progression, all of which are commonly observed in posttraumatic $\mathrm{OA}^{37,43}$. Size distributions obtained by direct conversion of SS-nanopore ECD measurements to MW for the same samples also showed a notable shift in the same direction (Fig. $4 \mathrm{~b}$, c), with greater resolution at the lower MW range $(<500 \mathrm{kDa})$ as compared to gels.

A second subject (H2) demonstrated an opposite shift toward larger MW after post-traumatic OA induction, as determined by gel analysis (Fig. 4d, e, insets). While OA is known to typically reduce mean HA size through mechanisms that may include joint friction shearing, enzymatic regulation, and immunological degradation, this effect could in principle be overshadowed by an upregulation of HA synthesis pathways during the acute posttraumatic phase ${ }^{44}$ or be affected by natural HA turnover to produce a net increase in MW. This sample provided an experimental counterpoint for our SS-nanopore validation. Indeed, from SS-nanopore size distribution analysis of $\mathrm{H} 2$ (Fig. 4d, e), we observed a notable shift toward higher MW HA 12 days after surgical induction of post-traumatic OA, verifying the results from gel electrophoresis.

We found that the size distributions obtained for sham knees (arthroscopically examined contralateral knees in which no carpal chip was created) were not significantly different from Day 0 to Day 5 or 12 , or compared to each other (Fig. $4 \mathrm{~b}, \mathrm{~d}$ ). This data illustrate the consistency of the measurement across samples and devices. We note that due to the size resolution of the initial SSnanopore analysis (c.f. Fig. 3d), it is possible that our distribution results overestimate the lowest MW HA in the detectable range and may miss extremely low-MW molecules entirely. This limitation can be improved in future iterations of the system. However, the collective data from the two equine synovial fluid samples presented here are compelling demonstrations of the efficacy of the approach for translational size analysis of HA from biological specimens.

\section{Discussion}

We have presented a SS-nanopore approach for the assessment of the glycan HA, an emerging biomarker with relevance to a broad range of diseases ${ }^{45}$. Through analysis of translocation properties, HA MW can be determined on a per molecule basis, eventually yielding overall size distribution from only a few hundred or thousand individual events. After showing that the platform could detect HA and demonstrating a general capacity to distinguish broad changes in its size distribution, we measured a consistent dependence of event ECD on HA MW using controlled quasi-monodisperse samples. Finally, we developed a general upstream isolation protocol for the specific isolation of HA from biological fluids toward the purpose of SS-nanopore HA size distribution determination in synovial fluid from an equine post-traumatic OA model. Such a sample in our prototype device consisted of as little as $10 \mathrm{ng}$ of $\mathrm{HA}$ in a $10 \mu \mathrm{l}$ volume, which could be measured electrically in $\sim 2 \mathrm{~h}$. This time could be shortened significantly by using higher HA concentrations.

This study establishes SS-nanopores as a tool for the analysis of HA, demonstrating high quality, reliable, and reproducible (Supplementary Figures 7-9) quantitative data on both HA detection and size distribution determination from biological specimens. The sensitivity, speed, and small sample volume requirements of this approach make it attractive as the basis for future diagnostic tools with distinct advantages over conventional glycan assessment technologies. Applications for the technology may include both translational measurement of $\mathrm{HA}$ as a biomarker, as well as assessment of HA synthesis products for commercial or research purposes. The results also suggest a wider role for the measurement platform in assessing other important glycans, GAGs, and proteoglycans that may have additional importance as bioindicators of diverse pathologies, including heparan sulfate ${ }^{46}$, chondroitin sulfate ${ }^{47}$, and keratan sulfate ${ }^{48}$.

\section{Methods}

HA samples. Purified polydisperse Streptococcus zooepidemicus HA (Vesta, Indianapolis, IN) was mixed as received in deionized water to a concentration of 1 $\mu \mathrm{g} / \mu \mathrm{l}$ as a bulk solution; no further purification was performed. Discrete quasimonodisperse HA samples ${ }^{33}$ were provided by Hyalose, LLC. (Oklahoma City, OK). A total of seven quasi-monodisperse HA samples $(54,81,130,237,545,1076$, and $2384 \mathrm{kDa}$ ) were used, with MW within $5 \%$ of the reported mean (polydispersity $=1.001-1.035$, as estimated by MALLS-SEC). Each $50 \mu \mathrm{g}$ lyophilized sample was mixed with deionized water to produce a $1 \mu \mathrm{g} / \mu \mathrm{l}$ solution. All samples 
were stored in LoBind Eppendorf tubes (Fisher Scientific, Hampton, NH) at $4{ }^{\circ} \mathrm{C}$ for short term use, or kept at $-20^{\circ} \mathrm{C}$ for long-term storage.

Ultrasonic shearing of polydisperse HA. A $50 \mu$ solution of polydisperse HA concentrate $(1 \mu \mathrm{g} / \mu \mathrm{l})$ was placed in a microTUBE AFA fiber snap-cap (Covaris, Woburn, MA) and mechanically sheared in a $7^{\circ} \mathrm{C}$ water bath using a Covaris $\mathrm{S} 220$ focused ultrasonicator (peak incident power of $175 \mathrm{~W}, 200$ cycles per burst, $10 \%$ duty factor). Shearing was varied by increasing sonication times in $5 \mathrm{~s}$ increments. HA fragmentation was monitored by gel electrophoresis using the methods described below.

Gel electrophoresis of HA. Electrophoresis was conducted on a $0.5 \%$ agarose gel in $1 \times$ TAE buffer. All samples (polydisperse and quasi-monodisperse HA) were aliquoted as $12 \mu \mathrm{l}$ volumes in $0.15 \mathrm{NaCl}$ solution using a minimum of $1-3 \mu \mathrm{g} \mathrm{HA}$ for visualization, consistent with previous literature ${ }^{49,50}$. For synovial fluid samples, collected material was centrifuged at $300 \times g$ for $5 \mathrm{~min}$ at $4{ }^{\circ} \mathrm{C}$ to pellet the cellular material, and the supernatant was retrieved and stored at $-80^{\circ} \mathrm{C}$. Prior to gel electrophoresis, the solution was thawed, diluted 1:20 in PBS buffer, and incubated with proteinase $\mathrm{K}(1 \mathrm{mg} / \mathrm{mL})$ overnight to digest protein components. The resulting mixture was loaded directly onto gel because analyte visualization was insensitive to the trace background components. Electrophoresis was performed at $34 \mathrm{~V}$ for $3.5 \mathrm{~h}$ at room temperature for polydisperse and quasi-monodisperse HA samples, and at $50 \mathrm{~V}$ for $8 \mathrm{~h}$ at room temperature for synovial fluid HA samples. Staining was performed as described previously ${ }^{49}$. Briefly, the gel was submerged overnight in a room temperature bath of $0.005 \%$ Stains-All (Sigma-Aldrich, St. Louis, MO) in $50 \%$ ethanol, with care taken to prevent light exposure. Next, destaining was performed by incubating the gel in $10 \%$ ethanol for $8 \mathrm{~h}$, still in the dark. The bath was refreshed with clean solution at least twice during this time. Finally, the gel was removed and excess ethanol solution was removed manually by wicking with laboratory wipes. Gel images were collected under white light transillumination using a ChemiDoc XRS + system (BioRad, Hercules, CA) for Figs. 23 and a VersaDoc system (BioRad) for Fig. 4. Migration distance was determined via image analysis (Image ${ }^{51}$ ) by determining the distance from the bottom of the loading well for each band.

SS-nanopore preparation. Silicon chips $(4 \mathrm{~mm})$ with a thin, free-standing silicon nitride (SiN) membrane (8-2 $\mu \mathrm{m}$ with $25 \mathrm{~nm}$ thickness) were obtained commercially (Norcada, Inc. Alberta, Canada) for solid-state nanopore fabrication. Individual pores were formed in-house using an Orion Plus helium ion microscope (Carl Zeiss, Peabody, MA) following methods described elsewhere ${ }^{52}$. Briefly, the focus and astigmatism of a focused helium beam were first optimized on an area of the silicon chip near the suspended SiN window using point exposures. Then, the beam position was blanked, moved to the center of the SiN membrane, and exposed for a calibrated time to produce a single pore with reproducible dimensions. All nanopores used in this work were formed with diameters in the range of $6.5-8.6 \mathrm{~nm}$. Following fabrication, chips were stored in $50 \%$ ethanol solution prior to use. In preparation for measurement, each nanopore chip was rinsed with DI water and absolute ethanol, then dried with filtered air, and subsequently exposed to a $30 \mathrm{~W}$ air plasma (Harrick Plasma, Ithaca, NY) for two minutes on each side before being positioned in a custom Ultem 1000 flow cell. Measurement buffer ( 6 $\mathrm{M} \mathrm{LiCl}, 10 \mathrm{mM}$ Tris, $1 \mathrm{mM}$ EDTA, $\mathrm{pH}$ 8.0) was then introduced to both sides of the chip. Prior to use, prepared buffers were treated in an ultrasonic bath (FS20, Fisher Scientific) for $5 \mathrm{~min}$ and then passed through a $0.45 \mu \mathrm{m}$ syringe filter (Minisart NY25, Sartorius, Bohemia, NY) to remove contaminants and precipitates. $\mathrm{Ag} / \mathrm{AgCl}$ electrodes (Sigma-Aldrich, St. Louis, MO) were positioned in each chamber for voltage application and ionic current measurement using an Axopatch 200B patch clamp amplifier (Molecular Devices, Sunnyvale, CA). Each chip was pre-checked using clean measurement buffer to ensure a steady, low-noise baseline current with no spurious events and a linear current-voltage $(I-V)$ curve that verified SS-nanopore diameter in assessment buffer $(1 \mathrm{M} \mathrm{NaCl}, 10 \mathrm{mM}$ Tris, 1 mM EDTA) using an established model ${ }^{31}$ modified to incorporate empirical conductivity of high-concentration electrolytes in aqueous solution ${ }^{53}$. Pore diameters were stable, varying by $<1 \mathrm{~nm}$ over typical measurement times (Supplementary Figure 10)

SS-nanopore analysis of HA. Prior to HA analysis, the assessment buffer was exchanged for measurement buffer to maximize signal-to-noise ratio ${ }^{54}$. HA was loaded by pipetting $10-20 \mu \mathrm{l}$ into one flow cell chamber at a final concentration of $50 \mathrm{ng} / \mu \mathrm{l}$ unless otherwise noted. The data were collected at a rate of $200 \mathrm{kHz}$ with a four-pole Bessel filter designed to be $100 \mathrm{kHz}$, but actually corresponding ${ }^{55}$ to 57 $\mathrm{kHz}$. Analysis was performed using custom software, with which an additional 5 $\mathrm{kHz}$ low-pass filter was applied to all collected data. Each sample was tested in a series of trials at voltages ranging typically from $100-400 \mathrm{mV}$. Event threshold was defined as a deviation of at least five standard deviations $(5 \sigma)$ from baseline current with a duration between $25 \mu \mathrm{s}$ and $2.5 \mathrm{~ms}$. HA translocation was confirmed via recapture events ${ }^{24}$ (Supplementary Figure 1), as well as observation of voltagedependent reductions in event duration for quasi-monodisperse HA (Supplementary Figure 11). ECD was calculated for each deviation as the area encompassing the event ${ }^{28}$ by integrating the nanopore current for the duration of time it remained beyond the $5 \sigma$ threshold value. Event rates were determined from uninterrupted current traces of $3.2 \mathrm{~s}$ increments at a single condition. The standard deviation measured between increments was used as an indication of measurement error.

Equine model of osteoarthritis. Equine synovial fluid was obtained from healthy adult horses (2-5 years old) with radiographically normal carpal joints. Posttraumatic osteoarthritis was induced surgically through a carpal chip defect in one randomly assigned forelimb ${ }^{42}$. Briefly, an $8 \mathrm{~mm}$ osteochondral fragment was created in the dorsal rim of the radial carpal bone and left within the joint. The exposed subchondral bone was then debrided using an arthroburr to generate a 15 $\mathrm{mm}$ defect; the debris generated from the procedure was not removed from the synovial cavity. A sham operation (arthroscopic examination without carpal chip induction) was performed on the contralateral leg to serve as a control. Two weeks after the induced osteochondral fragmentation, without operative intervention, the horses were subjected to a $30 \mathrm{~min}$ treadmill/5 days per week training regimen to initiate post-traumatic osteoarthritis (OA), and synovial fluid samples were collected from both joints on Day 0 as well as Day 5 (H1) or 12 ( $\mathrm{H} 2)$ post-surgery. Samples were kept at $-80^{\circ} \mathrm{C}$ prior to use. All animal and tissue collecting protocols were approved by the Cornell University's Institutional Animal Care and Use Committee (Protocol Number: 2012-0097).

High-purity HA extraction from synovial fluid. HA was isolated from the equine synovial fluid using a protocol adapted from Yuan et al. ${ }^{50}$. Raw equine synovial fluid $(50 \mu \mathrm{l})$ was first incubated with $1.8 \mathrm{U} / \mathrm{mL}$ proteinase $\mathrm{K}$ (New England Biolabs, Ipswich, MA) for $15 \mathrm{~min}$ at $37^{\circ} \mathrm{C}$ to digest protein components, including those with HA-binding capacity. An equal volume of a phenol:chloroform:isoamyl alcohol (25:24:1 v/v/v, Fisher Scientific) was then added to the sample and mixed thoroughly before being centrifuged for $15 \mathrm{~min}$ at $14,000 \times \mathrm{g}$ in a Phase Lock Gel Tube (QuantaBio, Beverly, MA) to separate the aqueous HA from the organic component. This extraction process was repeated once using pure chloroform to remove residual phenol from the aqueous phase, which was found to adversely affect downstream protein function during the affinity-based purification steps.

In preparation for the isolation of pure $\mathrm{HA}$, first streptavidin magnetic beads (Dynabeads M-280, Invitrogen, Carlsbad, CA) at a concentration $10 \mathrm{mg} / \mathrm{mL}$ were washed three times with $1 \times$ PBS, $0.05 \%$ Tween by adding buffer, mixing gently, and aspirating under magnetic field, and then three times with $1 \times$ PBS only. After washing, $250 \mu \mathrm{l}$ of packed beads were resuspended in $50 \mu \mathrm{l}$ of $1 \times$ PBS. Then, $21 \mu \mathrm{l}$ of biotinylated versican G1 domain (bVG1, $1.23 \mu \mathrm{g} / \mu \mathrm{l}$, Echelon Biosciences, Salt Lake City, UT) was added directly to the beads, mixed, and incubated for $1 \mathrm{~h}$ at room temperature on a rocker. After incubation, the beads were washed three times with $150 \mu \mathrm{l} 1 \times \mathrm{PBS}$ to remove any unbound bVG1.

The bVG1-streptavidin beads were subsequently reconstituted with the solventextracted HA solution and incubated at room temperature for $24 \mathrm{~h}$ with gentle rocking. The sample was placed on a magnet to pull down the beads (with bound HA) and the supernatant was aspirated. The beads were washed three times with $1 \times$ PBS, after which deionized water was added to the sample to a final volume of $50 \mu \mathrm{l}$. To denature the bVG1 and release the bound HA, the sample was placed on a heating block at $95^{\circ} \mathrm{C}$ for $15 \mathrm{~min}$. Finally, the vial was placed on a magnet and the solution containing released, purified HA was removed and stored at $-20^{\circ} \mathrm{C}$ until use. Yield was determined through direct quantification of the isolate with an enzyme-linked immunosorbent assay kit (HA ELISA, Echelon Biosciences; Supplementary Table 2).

Data Availability. The authors declare that the data supporting the findings of this study are available within the paper and its Supplementary Information files.

Received: 11 October 2017 Accepted: 14 February 2018

Published online: 12 March 2018

\section{References}

1. Fraser, J. R. E., Laurent, T. C. \& Laurent, U. B. G. Hyaluronan: its nature, distribution, functions and turnover. J. Intern. Med. 242, 27-33 (1997).

2. Shirali, A. C. \& Goldstein, D. R. Activation of the innate immune system by the endogenous ligand hyaluronan. Curr. Opin. Organ Transplant. 13, 20-25 (2008).

3. Balazs, E. A., Watson, D., Duff, I. F. \& Roseman, S. Hyaluronic acid in synovial fluid. I. Molecular parameters of hyaluronic acid in normal and arthritis human fluids. Arthritis Rheum. 10, 357-376 (1967).

4. Lokeshwar, V. B., Öbek, C., Soloway, M. S. \& Block, N. L. Tumor-associated hyaluronic acid: a new sensitive and specific urine marker for bladder cancer. Cancer Res. 57, 773-777 (1997). 
5. Neuman, M. G., Cohen, L. B. \& Nanau, R. M. Hyaluronic acid as a noninvasive biomarker of liver fibrosis. Clin. Biochem. 49, 302-315 (2016).

6. Cowman, M. K., Lee, H.-G., Schwertfeger, K. L., McCarthy, J. B. \& Turley, E. A. The content and size of hyaluronan in biological fluids and tissues. Front. Immunol. 6, 261 (2015).

7. Nakamura, K. et al. High, but not low, molecular weight hyaluronan prevents T-cell-mediated liver injury by reducing proinflammatory cytokines in mice. J. Gastroenterol. 39, 346-354 (2004).

8. Noble, P. W., Lake, F. R., Henson, P. M. \& Riches, D. W. Hyaluronate activation of CD44 induces insulin-like growth factor-1 expression by a tumor necrosis factor-alpha-dependent mechanism in murine macrophages. J. Clin. Invest. 91, 2368-2377 (1993).

9. Rayahin, J. E., Buhrman, J. S., Zhang, Y., Koh, T. J. \& Gemeinhart, R. A. High and low molecular weight hyaluronic acid differentially influence macrophage activation. ACS Biomater. Sci. Eng. 1, 481-493 (2015).

10. Sasaki, Y. et al. Quantitative measurement of serum hyaluronic acid molecular weight in rheumatoid arthritis patients and the role of hyaluronidase. Int. J. Rheum. Dis. 14, 313-319 (2011).

11. Haserodt, S., Aytekin, M. \& Dweik, R. A. A comparison of the sensitivity, specificity, and molecular weight accuracy of three different commercially available Hyaluronan ELISA-like assays. Glycobiology 21, 175-183 (2011)

12. Hokputsa, S., Jumel, K., Alexander, C. \& Harding, S. E. A comparison of molecular mass determination of hyaluronic acid using SEC/MALLS and sedimentation equilibrium. Eur. Biophys. J. 32, 450-456 (2003)

13. Yeung, B. \& Marecak, D. Molecular weight determination of hyaluronic acid by gel filtration chromatography coupled to matrix-assisted laser desorption ionization mass spectrometry. J. Chromatogr. A 852, 573-581 (1999).

14. Kuhn, A. V., Raith, K., Sauerland, V. \& Neubert, R. H. H. Quantification of hyaluronic acid fragments in pharmaceutical formulations using LC-ESI-MS. J. Pharm. Biomed. Anal. 30, 1531-1537 (2003).

15. Volpi, N. On-line HPLC/ESI-MS separation and characterization of hyaluronan oligosaccharides from 2-mers to 40-mers. Anal. Chem. 79, 6390-6397 (2007).

16. Bhilocha, S. et al. Agarose and polyacrylamide gel electrophoresis methods for molecular mass analysis of 5- to 500-kDa hyaluronan. Anal. Biochem 417, 41-49 (2011).

17. Cowman, M. K. et al. Improved agarose gel electrophoresis method and molecular mass calculation for high molecular mass hyaluronan. Anal. Biochem. 417, 50-56 (2011).

18. Li, J. et al. Ion-beam sculpting at nanometre length scales. Nature $\mathbf{4 1 2}$, 166-169 (2001).

19. Storm, A. J., Chen, J. H., Ling, X. S., Zandbergen, H. W. \& Dekker, C. Fabrication of solid-state nanopores with single-nanometre precision. Nat. Mater. 2, 537-540 (2003).

20. Fennouri, A. et al. Single molecule detection of glycosaminoglycan hyaluronic acid oligosaccharides and depolymerization enzyme activity using a protein nanopore. ACS Nano 6, 9672-9678 (2012)

21. Fennouri, A. et al. Kinetics of enzymatic degradation of high molecular weight polysaccharides through a nanopore: experiments and data-modeling. Anal. Chem. 85, 8488-8492 (2013)

22. Gatej, I., Popa, M. \& Rinaudo, M. Role of the $\mathrm{pH}$ on hyaluronan behavior in aqueous solution. Biomacromolecules 6, 61-67 (2005)

23. Firnkes, M., Pedone, D., Knezevic, J., Doeblinger, M. \& Rant, U. Electrically facilitated translocations of proteins through Silicon Nitride nanopores: conjoint and competitive action of diffusion, electrophoresis, and electroosmosis. Nano Lett. 10, 2162-2167 (2010).

24. Gershow, M. \& Golovchenko, J. A. Recapturing and trapping single molecules with a solid-state nanopore. Nat. Nanotechnol. 2, 775-779 (2007)

25. Li, J. L., Gershow, M., Stein, D., Brandin, E. \& Golovchenko, J. A. DNA molecules and configurations in a solid-state nanopore microscope. Nat. Mater. 2, 611-615 (2003).

26. Storm, A. J., Chen, J. H., Zandbergen, H. W. \& Dekker, C. Translocation of double-strand DNA through a silicon oxide nanopore. Phys. Rev. E 71, 051903 (2005).

27. Storm, A. J. et al. Fast DNA translocation through a solid-state nanopore. Nano Lett. 5, 1193-1197 (2005).

28. Fologea, D. et al. Detecting single stranded DNA with a solid state nanopore. Nano Lett. 5, 1905-1909 (2005).

29. Wanunu, M., Morrison, W., Rabin, Y., Grosberg, A. Y. \& Meller, A. Electrostatic focusing of unlabelled DNA into nanoscale pores using a salt gradient. Nat. Nanotechnol. 5, 160-165 (2010).

30. Grosberg, A. Y. \& Rabin, Y. DNA capture into a nanopore: interplay of diffusion and electrohydrodynamics. J. Chem. Phys. 133, 165102 (2010).

31. Wanunu, M. et al. Rapid electronic detection of probe-specific microRNAs using thin nanopore sensors. Nat. Nanotechnol. 5, 807-814 (2010).
32. Zahid, O. K., Zhao, B. S., He, C. \& Hall, A. R. Quantifying mammalian genomic DNA hydroxymethylcytosine content using solid-state nanopores. Sci. Rep. 6, 29565 (2016).

33. Jing, W. \& DeAngelis, P. L. Synchronized chemoenzymatic synthesis of monodisperse hyaluronan polymers. J. Biol. Chem. 279, 42345-42349 (2004)

34. Rosenstein, J. K., Wanunu, M., Merchant, C. A., Drndic, M. \& Shepard, K. L. Integrated nanopore sensing platform with sub-microsecond temporal resolution. Nat. Methods 9, 487-U112 (2012).

35. Ferrari, M. \& Bloomfield, V. Scattering and diffusion of mononucleosomal DNA - effects of counterion valence and salt and DNA concentration. Macromolecules 25, 5266-5276 (1992).

36. Litwiniuk, M., Krejner, A., Speyrer, M. S., Gauto, A. R. \& Grzela, T. Hyaluronic acid in inflammation and tissue regeneration. Wounds Compend. Clin. Res. Pract. 28, 78-88 (2016).

37. Band, P. A. et al. Hyaluronan molecular weight distribution is associated with the risk of knee osteoarthritis progression. Osteoarthr. Cartil. 23, 70-76 (2015).

38. Hui, A. Y., McCarty, W. J., Masuda, K., Firestein, G. S. \& Sah, R. L. A systems biology approach to synovial joint lubrication in health, injury, and disease. Wiley Interdiscip. Rev. Syst. Biol. Med. 4, 15-37 (2012).

39. Seyfried, N. T. et al. Expression and purification of functionally active hyaluronan-binding domains from human cartilage link protein, aggrecan and versican: formation of ternary complexes with defined hyaluronan oligosaccharides. J. Biol. Chem. 280, 5435-5448 (2005).

40. Day, A. J. \& Prestwich, G. D. Hyaluronan-binding proteins: tying up the giant. J. Biol. Chem. 277, 4585-4588 (2002).

41. Barker, S., Hawkins, C. \& Hewins, M. Mucopolysaccharides in Synovial fluid - detection of chondroitin sulphate. Ann. Rheum. Dis. 25, 209-213 (1966).

42. McIlwraith, C. W., Frisbie, D. D. \& Kawcak, C. E. The horse as a model of naturally occurring osteoarthritis. Bone Jt. Res. 1, 297-309 (2012).

43. Temple-Wong, M. M. et al. Hyaluronan concentration and size distribution in human knee synovial fluid: variations with age and cartilage degeneration. Arthritis Res. Ther. 18, 18 (2016).

44. Chan, D. D. et al. Deficiency of Hyaluronan Synthase 1 (Has1) results in chronic joint inflammation and widespread intra-articular fibrosis in a murine model of knee joint cartilage damage. Osteoarthr. Cartil. OARS Osteoarthr. Res. Soc. 23, 1879-1889 (2015).

45. Laurent, T. C., Laurent, U. B. \& Fraser, J. R. Serum hyaluronan as a disease marker. Ann. Med. 28, 241-253 (1996).

46. Tomatsu, S. et al. Dermatan sulfate and heparan sulfate as a biomarker for mucopolysaccharidosis I. J. Inherit. Metab. Dis. 33, 141-150 (2010).

47. Hayes, A. J., Tudor, D., Nowell, M. A., Caterson, B. \& Hughes, C. E. Chondroitin sulfate sulfation motifs as putative biomarkers for isolation of articular cartilage progenitor cells. J. Histochem. Cytochem. 56, 125-138 (2008)

48. Kubaski, F. et al. Di-sulfated keratan sulfate as a novel biomarker for mucopolysaccharidosis IVA. Mol. Genet. Metab. 114, S66-S67 (2015).

49. Lee, H. G. \& Cowman, M. K. An agarose gel electrophoretic method for analysis of hyaluronan molecular weight distribution. Anal. Biochem. 219, 278-287 (1994).

50. Yuan, H., Amin, R., Ye, X., De La Motte, C. A. \& Cowman, M. K. Determination of hyaluronan molecular mass distribution in human breast milk. Anal. Biochem. 474, 78-88 (2015).

51. Collins, T. J. ImageJ for microscopy. Biotechniques 43, 25-30 (2007).

52. Yang, J. et al. Rapid and precise scanning helium ion microscope milling of solid-state nanopores for biomolecule detection. Nanotechnology 22, 285310 (2011).

53. Haynes, W. M. CRC Handbook of Chemistry and Physics, 97th Edition. (CRC Press, Boca Raton, FL, 2016)

54. Kowalczyk, S. W., Wells, D. B., Aksimentiev, A. \& Dekker, C. Slowing down DNA translocation through a nanopore in lithium chloride. Nano Lett. 12, 1038-1044 (2012)

55. Uram, J. D., Ke, K. \& Mayer, M. Noise and bandwidth of current recordings from submicrometer pores and nanopores. ACS Nano 2, 857-872 (2008).

\section{Acknowledgements}

The authors wish to acknowledge Courtney Smith for early contributions to the measurements and Glenn Prestwich for helpful discussions. This work was supported by start-up funds to E.R. and A.R.H. from Wake Forest University Health Sciences.

\section{Author contributions}

F.R. performed biochemical treatments, handled physiological fluid samples, performed SS-nanopore measurements, and analyzed data. O.K.Z. contributed to experimental design and performed initial SS-nanopore measurements. H.L.R., B.T.P., and A.J.N. collected synovial fluid samples and performed gel analyses. P.L.D., E.R., and A.S. provided materials, contributed to experimental design, and edited the manuscript. E.R. and A.R.H. oversaw the project, contributed to data analysis, and wrote the manuscript. All authors reviewed the manuscript. 


\section{Additional information}

Supplementary Information accompanies this paper at https://doi.org/10.1038/s41467018-03439-x.

Competing interests: A.R.H, E.R., and P.L.D. are listed as inventors on a provisional patent involving the described technology. Remaining authors declare no competing interest.

Reprints and permission information is available online at http://npg.nature.com/ reprintsandpermissions/

Publisher's note: Springer Nature remains neutral with regard to jurisdictional claims in published maps and institutional affiliations. (c) (i) Open Access This article is licensed under a Creative Commons Attribution 4.0 International License, which permits use, sharing, adaptation, distribution and reproduction in any medium or format, as long as you give appropriate credit to the original author(s) and the source, provide a link to the Creative Commons license, and indicate if changes were made. The images or other third party material in this article are included in the article's Creative Commons license, unless indicated otherwise in a credit line to the material. If material is not included in the article's Creative Commons license and your intended use is not permitted by statutory regulation or exceeds the permitted use, you will need to obtain permission directly from the copyright holder. To view a copy of this license, visit http://creativecommons.org/ licenses/by/4.0/.

(C) The Author(s) 2018 